\title{
Optimization of Machine Flexibility in an Ion Plating Cell
}

\author{
F. T. S. Chan, K. C. Au and P. L. Y. Chan \\ Department of Industrial and Manufacturing Systems Engineering, The University of Hong Kong, Hong Kong
}

\begin{abstract}
In 1970s, manufacturing system performance was heavily depended on productivity. Manufacturers only concentrated on increasing productivity by increasing the number of workforce. The concept of flexibility began to introduce into the industry since 1990s, manufacturers realized that flexibility was a better solution to improve productivity by responding the changing environment in manufacturing system. However, limited researches on machine flexibility in lon Plating (IP) industry were studied, most of them have focused on product development and quality of coating. The aim of this paper is to determine the optimal level of machine flexibility in an Ion Plating Cell (IPC) to improve the entire system performance. A Machine Loading Sequencing (MLS) model based on multi-objectives Genetic Algorithms (GA) was developed. In the case study, industrial data of a precious metal finishing company has been input into the proposed Machine Loading Sequencing Genetic Algorithm (MLSGA) model. Different level of machine flexibility will be assigned into different machines to determine the optimum while the overall system performance (i.e. on-time delivery, quality of product and production cost) has been maximized. The results demonstrated that the machine flexibility level in IPC should be zero under the recent IP technology. However, when the quality of coating is improved, machine flexibility should be introduced.
\end{abstract}

Keywords-Ion plating, machine flexibility, machine loading, penalty, quality

\section{INTRODUCTION}

Flexibility can be defined as the ability to respond effectively to the changing environment. Generally, there are thirteen common types of flexibilities: machine, material handling, operations, process, product, routing, volume, expansion, program, production, material, labor and market [1]. As uncertainty can affect the effectiveness and efficiency of the entire manufacturing system, responsiveness on uncertainty has been concerned heavily by manufacturing industry in these few decades. While introducing machine flexibility into the system, improvements on system performance and responsiveness on uncertainty are expected. Hence, machine flexibility has been chosen as one of the major concerns in this study. Machine flexibility has been referred to the number and variety of operations that a machine can perform without incurring high transition penalties or large changes in performance outcomes [2].

In this study, the concept of machine flexibility was applied into IPC since limited researches on machine flexibility in IP industry were studied. IP is a manufacturing process used to deposit elements onto a substrate via a reactive gas. A negative charge is applied to the substrate to ionize the reactive gas. Coating material is vaporized into the glow discharge of the ionized gas. The coating vapor are ionized and accelerated toward the substrate surface where they will be adhered and form a coating. IP can use vacuum evaporation sputtering or arc vaporization as the source of the depositing material [3]. At recent, cost, quality, and delivery time are the major concerns of most of the organizations, system performance becomes one of their key dimensions to increase the competitive advantages. In order to maximize the level of system performance in IPC, the proposed MLSGA model has considered six criteria: number of tardy job(s), maximum tardiness time, number of early job(s), maximum earliness time, penalty on quality of coating and machine utilization index. Non-production time was being considered in order to improve the suitability of MLSGA model to the real circumstance in IPC.

In the rest of this paper, literature review on Machine Flexibility is stated in Section II. Then, a case study on application of MLSGA in an existing Metal Finishing Company to determine the optimal level of machine flexibility will be described in Section III. Results and discussions on the proposed model will be illustrated in Section IV. Conclusion of the entire study will be presented in Section V.

\section{LITERATURE REVIEW}

There are various types of flexibility have been defined by previous researchers that can affect the entire manufacturing systems significantly. Most of them tend to agree that flexibility can be beneficial to system performance, including shorter lead time, higher machine utilization and smaller in-process inventories etc. However, longer setup times, larger lot sizes, and longer material handling time, are generally omitted while concerning flexibility in their proposed models [4]. Among all different types of flexibility, reference [5] stated it was important to mention machine flexibility in manufacturing systems. The degree of machine flexibility can influence the level of system performance in five major ways, machine loading, part grouping, part ratio selection, tool loading, and scheduling in short-term. In the absence of operational penalties, such as setup times, 
increased pooling has been shown always to be beneficial to system performance. It was rare to have none operational penalty while considering machine flexibility in a manufacturing system. Operational penalty is a must to be considered in the system in order to cope with the reality [6].

As mentioned before, cost, quality, and delivery time are the major concerns of most of the manufacturers. While introducing machine flexibility into the systems, increase in competitive advantages can be expected by improving system performance and responsiveness on uncertainty, especially in IP industry. Hence, machine flexibility has been concerned in IPC to improve the overall system performance. In the proposed MLSGA model, inventory holding cost (i.e. number of early job(s)), quality of service (i.e. on-time delivery), quality of product, and production cost (i.e. machine utilization) would be considered to determine the optimal level of machine flexibility in IPC.

\section{CASE Study: Application of Machine Loading SEQUENCING GENETIC AlgORITHM (MLSGA)}

\section{A. Background}

A precious metal finishing company has been chosen that its major outputs are electroplating and ion plating products. Its customers are mainly in Watch \& Clock Industry. The case study was focused on optimizing the machine flexibility by using the model of MLSGA in the IPC of the company. There are three major types of coating; Ion Plating Gold (IPG), Ion Plating Stainless Steel (IPS), and Ion Plating Black (IPBK) are performed in the IPC. At present, the machine flexibility level of all the IP machines is zero, i.e. only one specific type of coating can be performed. When the next operation (e.g. IPS) is different to the previous operation (e.g. IPG), the non-production time (i.e. cleaning time, setup time, time for tuning color, etc.) of an IP machine between operations will be extremely long. According to the recent IP technology, the model is investigated with the assumption that the non-production time of IP machine is at an acceptable level. In order to improve the system performance of the IPC, the precious metal finishing company required MLSGA to act as a decision support tool to provide information to analyze their strategies and economic value to (i) minimize the inventory holding costs by reducing the number of early job(s) and maximum earliness time of all the available jobs; (ii) provide on-time delivery by reducing the number of lateness production; and (iii) minimizing the production unit cost by maximizing the machine utilization.

\section{B. Assumptions}

Some assumptions have been made as below: -

- Multiple machines are available for production in IPC.
- Each IP machines can perform all types of coating, i.e. IPG, IPS, and IPBK.

- All IP machines have the same production unit space available for production, i.e. fixed capacity.

- All jobs are available at time zero.

- Only one operation is required to perform on each received job.

- All available jobs have already been grouped into the batches with the best combination under the considerations on quality of product and due date of each job.

- Loading/unloading time is assumed as 15 minutes.

- Non-production times are incurred when an IP machine changes from one type of operation (e.g. IPG) to a different type of operation (e.g. IPS). The non-production times are predefined as 30 minutes which including cleaning time, setup time, time for tuning color etc., while loading/unloading time for workpieces will be considered separately.

- For all IP machines, when next operation is same as the previous operation, only loading/unloading time for workpieces will be considered. Nonproduction time will be assumed to be zero.

\section{Objective Function}

In this case study, multi-objective Genetic Algorithm have been applied in MLSGA model, which the objective function has considered inventory holding cost, on-time delivery, quality of product, and production cost. These four criteria could be used to measure the system performance in order to provide decision support information to analyze their strategies. The equation below was the objective function defined by integrating the real situation in IPC and experience from Production Manager of the company.

$$
\operatorname{Min} \sum w_{t n} T n+w_{t t} T t+w_{e n} E n+w_{e t} E t+w_{p e} P e-w_{u} U
$$

$$
\begin{array}{cll}
\begin{array}{c}
\text { where } \\
\mathrm{Tn}
\end{array} & - \text { Number of tardy job(s) } \\
\mathrm{Tt} & - & \text { Maximum tardiness time among all available } \\
& & \text { batches } \\
\mathrm{En} & - & \text { Number of early job(s) } \\
\mathrm{Et} & - & \text { Maximum earliness time among all available } \\
& & \text { batches } \\
\mathrm{Pe} & - & \text { Total penalty values for all available batches } \\
\mathrm{U} & - & \text { Machine utilization index } \\
w_{t n} & - & \text { Weight of parameter Tn } \\
w_{t t} & - & \text { Weight of parameter } \mathrm{Tt} \\
w_{e n} & - & \text { Weight of parameter En } \\
w_{e t} & - \text { Weight of parameter } \mathrm{Et} \\
w_{p e} & - \text { Weight of parameter Pe } \\
w_{u} & - & \text { Weight of parameter } \mathrm{U} \\
\text { and } w_{t n} & +w_{t t}+w_{e n}+w_{e t}+w_{p e}+w_{u}=1
\end{array}
$$


The weights of each criterion shown in (1) indicated their relative importance of the particular criteria which were predefined by Production Manager according to their experience. The more importance of the associated criterion, the larger the value of the weight. Detail illustration on each criterion is stated below.

1) Number of tardy job(s), Tn

In the last decade, providing better quality of product and on-time delivery are the minimum customer requirements for a company to be achieved. Late delivery of job can lead to complain on dissatisfying their requirements and may result in potential loss of reputation or changing supplier. In order to achieve higher level of customer satisfaction, the system performance should be maximized by improving the efficiency of the system, such that the production lead time of all available jobs could be reduced. According to these concerns on system performance, the number of tardy job(s) has been considered in the model to determine the level of system performance. The number of tardy job(s) is defined by the number of late delivery for all available jobs in this model. The less the number of tardy job(s), the higher the level of system performance can be achieved.

2) Maximum tardiness time, $T t$

Time to market is one of the critical points to decide the success of product. When the production lead time has been prolonged, it can result in product failure. On-time delivery has become the major concern for customer to make decision on placing order. Once the order has happened to late delivery, the length of tardiness time would be important for customer to consider. The shorter the tardiness time of the order, the lower the level of customer dissatisfaction. In this case study, tardiness time is calculated based on the time between the date of late delivery and the due date for each job. Maximum tardiness time is the longest tardiness time required among all the tardiness time of each available job. In order to minimize the tardiness time to satisfy customers, the production efficiency should be maximized to produce jobs with the shortest time. Hence, the lower the value for maximum tardiness time can be achieved by the company, the higher the level of system performance in IPC.

3) Number of early job(s), En

Early job is defined as job completed before its due date requested by customer. The concept of "Just-intime" (JIT) is introduced into the manufacturing industry since the mid 1990s. Customers are not expected to receive products before or after their requested delivery date. Most of the companies will tend to complete before the due date of the job in order to satisfy the customer requirements. However, the finished products have to hold in inventory until the delivery date, inventory holding cost will be incurred as earliness penalty. As cost is one of the major measurements to determine the level of system performance, costs should be minimized as possible to increase the profit margin of a company. In this model, the objective function would tend to minimize the number of early job(s), so it can increase the level of system performance by reducing the inventory holding cost due to the early jobs.

4) Maximum earliness time, Et

As mentioned previously, when a job is finished before its due date, it would be stored as inventory until its due date and incurs an earliness penalty in additional inventory holding cost. In relation to this concern, the additional inventory holding cost of the job is relatively correlated with the length of the earliness time. The longer the length of earliness time, the higher the inventory holding cost. Maximum earliness time is defined the longest earliness time required among all the earliness time of each available job. Hence, this model would minimize the maximum earliness time to reduce the additional inventory holding cost, such that the level of system performance can be improved.

5) Total penalty values for all available batches, $P e$ At present, IP has been widely applied in the manufacturing industry which provides tremendous advantages on prolonging product lifetime, reducing production cost and so on. However, IP technology is still in the developing stage, the quality of coating is highly depended on the performance of individual IP machine. In order to suit the MLSGA with the real world application, penalty on quality of coating has been introduced into the objective function to indicate the significant differences for products produced by different IP machine. The calculation on total penalty value for all available batches has been defined as shown in (2).

$$
P e=\sum_{i=1}^{n} r_{i j}
$$

\footnotetext{
where

$r_{i j}$ - Penalty rating of batch i while the selected machine is in the $j$ th priority

$n$ - Total number of batches have been included in the proposed model
}

Each job would have its corresponding penalty ratings on its selected IP machines. These ratings would be predetermined by the Production Manager of the company based on the performance of the IP machines in their IPC. When a batch is produced by its first priority machine, the penalty rating will define as zero since the batch will be produced by the best machine. For the measurement of non-production time, while a production batch required a machine to change from one type of coating to a different type of coating, non-production times will be included. Based on the recent technology of IP, the non-production time is extremely long, such that 30 minutes has been 
assumed as the non-production time required to configure the machine. In this model, the total penalty value should be minimized to achieve better quality of coating such that higher level of customer satisfaction will be expected.

6) Machine utilization index, $U$

According to the IP technology, hundreds of products are being produced simultaneously in an IP machine, and the amount of raw materials that used to produce a unit of product or hundred of products simultaneously, is the same. It indicates that the amount of materials being consumed is not correlated to the number of products being placed in the IP machine during the production. Although fully utilization of all possible space-unit inside the IP machine could minimize the production unit cost, there was a capacity limit on number of product for production in order to control the quality of coating. As minimizing production cost was one of the major objectives of the proposed MLSGA model, maximizing the utilization of machine could lead to minimize production unit cost under the technology nature of IP. Machine utilization index is calculated based on the production sequence of batches in their corresponding machines which the equation has shown in (3)

$$
U=\sum_{i=1}^{n} \sum_{j=1}^{m_{i}}\left(1-\frac{j-1}{m_{i}}\right) u_{i j}
$$

where

$u_{i j}$ - Machine utilization of the batch in the $j$ th production sequence of machine $i$

$m_{i}$ - Frequency of machining operations required machine $i$ to complete all available batch

In order to minimize the production cost, the batch with the higher machine utilization will tend to give a higher priority to process in its corresponding machine. The higher the machine utilization index, the lower the production cost in IPC.

\section{E. Procedures}

For the application of MLSGA, a formalized database has been developed to gather all relevant data (i.e. batch specifications, machine specifications, production time, etc.) for executing the model. MLSGA was acted as a decision support tool to provide information to determine the optimal machine flexibility level of each machine in IPC. It will be executed by assigning different level of machine flexibility into different machines by simply run through the procedures which are initialization, calculation of fitness, selection, crossover and mutation to determine the level of system performance under different combination of machine flexibility level. In this paper, a real dataset has been collected from the IPC and simulations of the proposed MLSGA are executed. A set of computational results are compared with each other and stated in the following section.

\section{RESUlTS AND DISCUSSION}

The weighting of each criterion of the objective function is defined by Production Manager under their recent circumstance. The proposed MLSGA simulates the machine loading schedule in the IPC which the criteria has been defined as $w_{t t}=w_{t n}=0.15, w_{e t}=w_{e n}=0.05$, and $w_{p e}=w_{u}=0.3$. Different level of machine flexibility have been assigned into different IP machines to execute the proposed model to generate a set of computational results which the weights of each criterion are fixed. The set of results are analyzed in order to determine the optimal level of machine flexibility in the IPC. The dataset that used for execution is the industrial dataset provided by the company. A total of 22 batches (i.e. consist of 50 jobs) are included in this dataset, and all the jobs are available on the same working day (i.e. Day 1). Each job has different manufacturing specifications, and with different quantity of products required to process.

For the simulation of the proposed MLSGA, there are several parameters required to be pre-set: (i) Population size $=500$; (ii) Number of generations $=3000$; and (iii) Number of IP machines available in IPC $=3$ (i.e. M1; M2 and M3). Totally 27 combinations can be formed with 3 different types of coating in this case study. Table I shows the critical computational results of MLSGA model, five different combinations of machine flexibility level in IPC are chosen to be discussed. Each of them can represent the effectiveness of different level of machine flexibility. Trial 1 (see Table I) shows the system performance of IPC while the machine flexibility level of all three available machines are zero (i.e. each machine can only

TABLE I

COMPUTATIONAL RESULTS OF THE PROPOSED MLSGA MODEL

\begin{tabular}{|c|c|c|c|c|c|c|c|c|c|c|}
\hline Trial no & $\begin{array}{c}\text { Machine } \\
\text { flexibility } \\
\text { level of M1 }\end{array}$ & $\begin{array}{c}\text { Machine } \\
\text { flexibility } \\
\text { level of M2 }\end{array}$ & $\begin{array}{c}\text { Machine } \\
\text { flexibility } \\
\text { level of M3 }\end{array}$ & $\begin{array}{c}\text { Fitness } \\
\text { value }\end{array}$ & $\begin{array}{c}\text { No. of } \\
\text { tardy job, } \\
\text { Tn }\end{array}$ & $\begin{array}{c}\text { Maximum } \\
\text { tardiness } \\
\text { time, Tt }\end{array}$ & $\begin{array}{c}\text { No. of } \\
\text { early job, } \\
\text { En }\end{array}$ & $\begin{array}{c}\text { Maximum } \\
\text { earliness } \\
\text { time, Et }\end{array}$ & $\begin{array}{c}\text { Total } \\
\text { penalty } \\
\text { values, Pe }\end{array}$ & $\begin{array}{c}\text { Machine } \\
\text { Utilization } \\
\text { index, } U\end{array}$ \\
\hline 1 & 0 & 0 & 0 & 1.5642 & 0 & 0 & 42 & 6 & 0 & 2.7859 \\
\hline 2 & 1 & 0 & 0 & 7.3017 & 0 & 0 & 38 & 6 & 20 & 2.9945 \\
\hline 3 & 0 & 0 & 1 & 7.5038 & 0 & 0 & 43 & 5 & 20 & 2.9875 \\
\hline 4 & 1 & 1 & 1 & 8.7566 & 0 & 0 & 38 & 5 & 25 & 2.978 \\
\hline 5 & 2 & 2 & 2 & 8.8066 & 0 & 0 & 39 & 5 & 25 & 2.978 \\
\hline
\end{tabular}


perform one type of coating). For Trial 2, 3 and 4, they represent the results of machine flexibility level is one which is the machine can perform at most two types of coating. Trial 5 shows the result of machine flexibility level is equal to two which implies all machines can perform three different types of coating. Under this circumstance, the lowest fitness value among all different combinations of machine flexibility level is $\mathbf{1 . 5 6 4 2 .}$

While comparing the computational results shown in Table I, Trial 2 and 4 have the least number of early jobs (i.e. 38), lesser inventory holding cost can be expected. Trial 3,4 and 5 have a lower value of maximum earliness time, it indicates that lower additional inventory holding cost will be required. Between these two criteria (number of early job and maximum earliness time), it implies that Trial 4 has the relatively lower overall inventory holding cost among all the other trials. The total penalty values of Trial 2, 3, 4 and 5 are more than 20, only Trial 1 does not have any penalty during production in IPC. Under the issue of production cost, the machine utilization index of most of the trials is around 2.9 , only Trial 1 is relatively low (i.e. 2.7859). In the MLSGA model, the objective function has been included all of the six mentioned criteria which each of them has different level of impact to the system performance while considering machine flexibility in the cell. The fitness value is the result of objective function which indicates Trial 1 has the lowest fitness value among all trials. It demonstrated that machine flexibility level should be assigned to zero in order to maximize the system performance under the recent IP technology. As mentioned previously, Trial 1 has relatively low in machine utilization index, high in the number of early jobs and maximum earliness time while comparing with all the other trials. However, no penalty value would be expected when the combination of Trial 1 is arranged in the cell. Quality is the most concerned issue in the recent IP industry, the criteria of quality has been placed with a relatively high weight in the objective function of MLSGA model. Quality of coating is highly depended on the performance of a particular IP machine in the recent IP technology. Once the machine flexibility has been introduced into the IPC, the product quality will be affected significantly (i.e. high in penalty value) when a particular coating is not processed in its best performed IP machine. Although the quality is important to the system performance in IPC, the performance of an IP machine to produce different type of coating can be improved based on the development of IP technology. Hence, machine flexibility should be introduced into the IPC when the IP technology is reached its mature stage. Once the quality of coating has been reached to a certain level, the MLSGA model should be simulated under their circumstance again in order to determine the optimal level of machine flexibility in the IPC.

\section{CONCLUSION}

Upon the completion of this study, a highly integrated Decision Support System (DSS) Database, a computer model on machine loading sequencing (MLSGA) has been elaborated. By using the developed DSS, the optimal level of machine flexibility can be determined under the considerations on on-time delivery, quality of coating and production cost (machine utilization) in the system performance of the cell. In the case study, it has illustrated that introducing machine flexibility into the IPC could not improve the entire system performance significantly under the recent IP technology. However, it indicates that when quality of coating can be improved to a certain level, machine flexibility should be considered in the IPC in order to maximize the system performance by minimizing the overall earliness and tardiness delivery (i.e. reduction of inventory holding cost and on-time delivery), decreasing the penalty value (i.e. quality of product), and minimizing the production cost while arranging the batch with higher machine utilization in higher priority to process. Moreover, while considering machine flexibility in the proposed MLSGA, it has demonstrated the importance of taking into account the quality of product, quality of service and penalty which previous publications on machine loading problems have not been mentioned.

\section{REFERENCES}

[1] R. Narain, R. C. Yaday, J. Sarkis and J. J Cordeiro, "The strategic implications of flexibility in manufacturing systems," International Journal of Agile Management Systems, vol. 2, no. 3, pp. 202-213, 2000.

(2) A. Petroni and M. Bevilacqua, "Identifying manufacturing flexibility best practices in small and medium enterprises," International Journal of Operations and Production Management, vol. 22, no. 8, pp. 929-947, 2002.

[3] D. M. Mattox, "Physical Vapor Deposition (PVD) Processes," Metal Finishing, vol. 98, no. 1, pp. 410-423, 2000.

[4] S. Benjaafar, "Modeling and analysis of machine sharing in manufacturing systems," European Journal of Operational Research, vol. 91, issue 1, pp. 56-73, 1996.

[5] Z. M. Mohamed, M. A. Youssef, and F. Huq, "The impact of machine flexibility on the performance of flexible manufacturing systems," International Journal of Operations and Production Management, vol. 21, no. 5, pp. 707-727, 2001.

[6] M. Sheikhzadeh, S. Benjaafar, and D. Gupta, "Machine sharing in manufacturing systems: total flexibility versus chaining," International Journal of Flexible Manufacturing Systems, vol. 10, issue 4, pp. 351-378, 1998. 